\title{
Editorial \\ Desafío presupuestario para las revistas científicas de la Universidad del Zulia
}

Se presenta en esta oportunidad el Número 65 de la Revista Venezolana de Gerencia correspondiente a los meses Enero-Marzo 2014. En este año que inicia, se vislumbran grandes desafíos relativos al tema presupuestario para la subvención de las revistas científicas de la Universidad del Zulia (LUZ) por parte del Consejo de Desarrollo Científico, Humanístico y Tecnológico de LUZ (organismo financiante). Aumentos en los presupuestos por el incremento de los costos de edición, impresión y distribución de la publicación, muestran la imposibilidad de cubrir dichas erogaciones con el presupuesto aprobado para este año 2014. Situación que hace cuestionar el futuro de las ediciones físicas de las revistas para este año, en el que se presenta como opción la necesidad de migrar hacia plataformas electrónicas que reduzcan los costos de edición y permitan seguir manteniendo al día nuestras revistas; con todas sus ediciones y con la calidad en cuanto a cientificidad y rigurosidad que las han caracterizado.

Esta opción de migrar hacia el formato de publicaciones electrónicas ha tomado auge en los últimos años. Gran cantidad de revistas que se editan hoy en día han asumido esta vía, demostrando que esto no tiene por qué afectar su proceso editorial, a la vez que han mostrado eficiencia en cuanto a tiempo y recursos empleados.

Queda de parte de los equipos editoriales en primera instancia vislumbrar la posibilidad de esta migración y minimizar su publicación física para lo que sea necesario (intercambios-canjes, indices, entre otros); y luego, de mayor importancia discutir acerca de las nuevas estrategias para la difusión de sus ediciones en formato electrónico y la reestructuración de los equipos de trabajo en virtud del nuevo formato de publicación, considerando que pareciera ser la opción más viable en razón de un presupuesto limitado y altos costos de impresión existentes en el mercado. Es en este caso, apostar a la búsqueda de la eficiencia en el uso de los recursos disponibles, sin dejar de lado los procesos que las han diferenciado de revistas similares en el área.

Luego de esta breve presentación de la situación actual y con noticias sobre el posible cambio en relación al formato de publicación de las revistas, no se puede dejar de lado la importancia de la publicación actual, que como siempre trae aportes de estudios nacionales e internacionales que enriquecen las áreas de investigación abordadas por cada uno.

Se presentan en esta oportunidad 8 artículos, a saber: Análisis de las relaciones entre la legitimidad organizativa, sus fuentes y dimensiones de la autoría de Cruz-Suárez, Ana; Díez-Martín, Francisco; Blanco-González, Alicia y Prado-Román, Camilo; seguido de Actitudes y engagement en el trabajo como antecedentes del comportamiento altruista de Álvarez Pérez, Dolores; Castro Casal, Carmen y Vila Vázquez, Guadalupe; ambos procedentes de Universidades españolas.

Desde México, Mendoza Ramírez, Luis y Toledo López, Argelia del Instituto Politécnico Nacional (IPN-CIIDIR) disertan acerca de la Orientación estratégica y desempeño de negocios artesanales afectados por crisis en Oaxaca, México; mientras que Aguilar Rivera, Noe, desde la Universidad Veracruzana estudia la Gestión de factores limitantes para la diversificación de la agroindustria azucarera. 
Editorial. Wileidys Artigas

Acompañándonos desde Chile; Araya-Castillo, Luis y Pedreros-Gajardo, Margarita de la de la Universidad Mayor de Chile y de la Consultora Value Creation Group respectivamente, colaboran con el trabajo Conformación de grupos estratégicos en sectores de educación superior; seguido de Vallejos-Romero, Arturo; Oyarzún Gutiérrez, Ivone y Garrido Castillo, Jaime desde la Universidad de la Frontera, con el artículo Salmonicultura 2.0 en Chile: una mirada desde la gobernanza ambiental.

Como séptimo artículo, desde Argentina Szlechter, Diego presenta La cultura corporativa: una revisión crítica desde la Sociología del trabajo; finalizando con el trabajo de Márquez, Marveya; Molina, Arturo y García, Juan de la Universidad de los Andes- Venezuela, intitulado: Orientación al mercado en la industria farmacéutica: influencia del poder y el conflicto sobre los resultados empresariales.

No queda mas que agradecer a los investigadores que han depositado la confianza en nuestras revistas e invitarlos a seguir enviando sus aportes, que, cualquiera sea el formato en de la publicación, lo importante es su contribución al desarrollo de la ciencia, siempre en constante evolución.

Dra. Wileidys Artigas

Directora de la Comisión de Estudios Humanísticos y Sociales del Consejo de Desarrollo Científico, Humanístico y Tecnológico (CONDES) Universidad del Zulia 